\title{
A qualitative analysis of psychological consultations for nurses working in a palliative care department (unit)
}

* Xinyi Liu, Yu Kawamata, Akiko Kawamoto, Yuka Mizuno, Yutaka Haramaki

Graduate School of Education, Hiroshima University, Higashihiroshima, Hiroshima, Japan

Contact: m183736@Hiroshima-u.ac.jp

What is palliative care like in the unit we (psychologists) worked for?

* It is comprised of a team involving doctors, nurses, pharmacists, social workers, volunteers, psychologists, and others.

Nurses typically spend the most time caring for patients and their families.

Nurses carry a lot of burden when caring for patients as a mediator between patients and their families.

What does the unit expect clinical psychologists to do?

Provide the following psychological consultation for nurses and other colleagues

Ways to communicate with patients or families undergoing difficult circumstances

To give mental support for nurses and others who feel stressed from work.

\section{What services do we provide in the palliative care unit?}

Providing psychological consultation for nurses and other colleagues (once a month)

Working in the department as a part-time job (once a week)

\section{Purpose}

\section{STUDY}

To specify the role of psychological consultations in helping nurses to solve their problems

\section{Methods}

Participants: 5 nurses working in a palliative care unit

Materials: Reports written by nurses after consultation

Methods: Labeling and categorizing was conducted using the $\mathrm{KJ}$ method

“KJ method : A generalized brainstorming technique ("idea-generating" methodology) to gather qualitative data which is originated and developed by a Japanese ethnologist (Scupin, 1997). We used this method firstly to separate nurses' reports into one-sentence comments, then to gather them into several categories, and finally to line up these categories to see the inner connection between them (Fig.1).

\section{Nurses' Worries}

communication with

patients and families (10)

Worries about their identity as a nurse (3)
Worries about

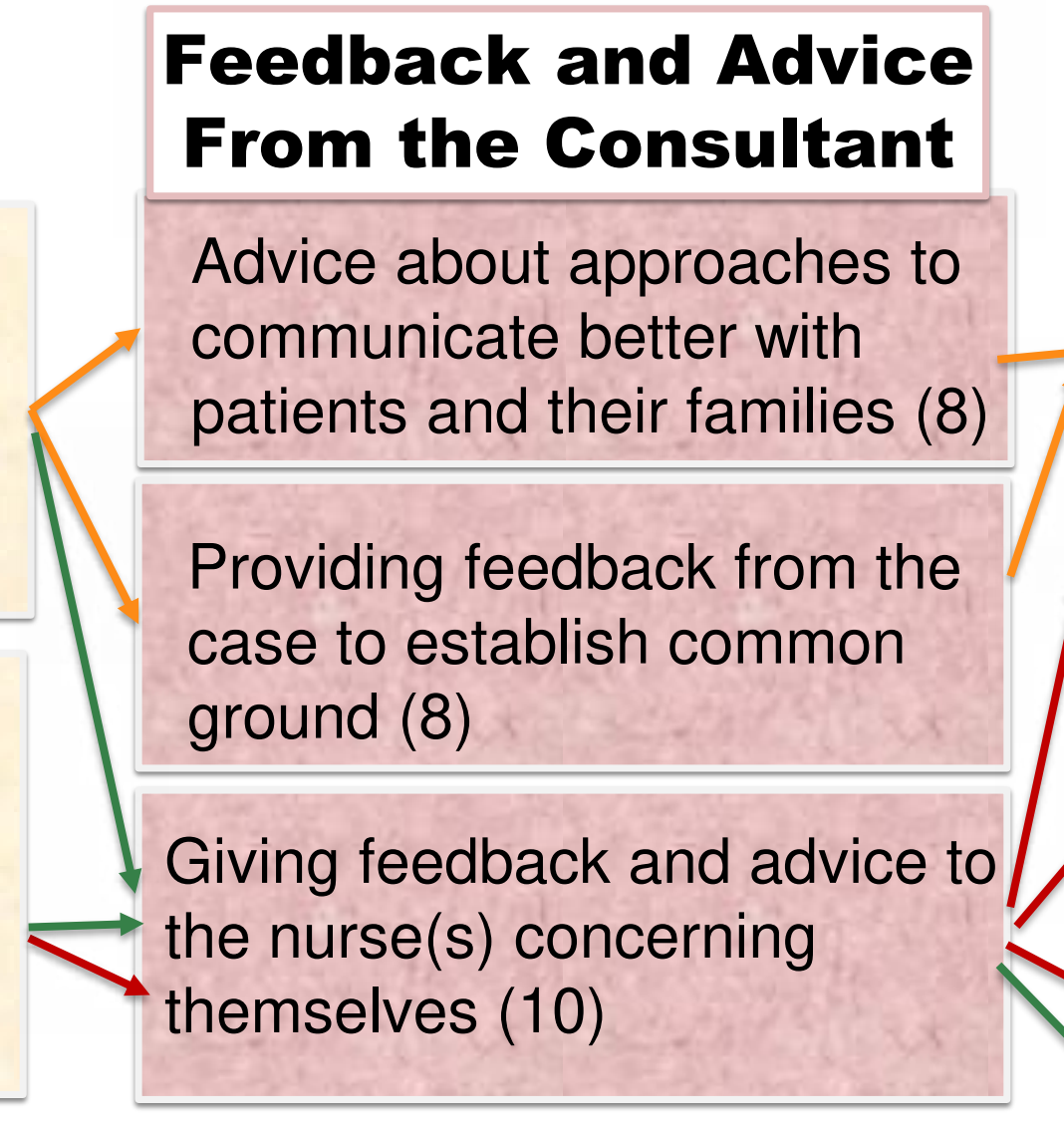

Feedback and Advice rom the Consultant

Advice about approaches to patients and their families (8)

Providing feedback from the Giving feedback and advice to the nurse(s) concerning mselves $(10)$

Fig. 1. Categories and their connections (number in parentheses refers to number of qualitative comments related to each category)

\section{Lessons and Insights From Consultation \\ Psychological knowledge from consultations (7) \\ Recollections of past behaviors and emotions (4) Realizations of new ideas from consultations (4) \\ Positive changes after consultations (5)}

